\title{
Demonstration of OPSORO - an Open Platform for Social Robots
}

\author{
Cesar Vandevelde \\ Dept. of Industrial Systems Engineering and Product \\ Design \\ Ghent University, Campus Kortrijk \\ Kortrijk, Belgium \\ Partner of Flanders Make \\ cesar.vandevelde@ugent.be
}

\author{
Jelle Saldien \\ Dept. of Industrial Systems Engineering and Product \\ Design \\ Ghent University, Campus Kortrijk \\ Kortrijk, Belgium \\ Partner of Flanders Make \\ jelle.saldien@ugent.be
}

\begin{abstract}
One of the major obstacles in the study of humanrobot interaction with social robots is the lack of platforms to allow for tests with large user groups. Often, the price of these robots prohibits using more than a handful of robots. Another factor is that with commercial platforms, the robots do not possess all the necessary features to perform an experiment and due to the closed nature of the platform, extensive modifications are nearly impossible. To address this problem, a new social robotics platform, OPSORO, is presented. The platform uses an innovative modular system design that enables the creation of different embodiments to represent anthropomorphic robots focusing on face-to-face communication. Our goal is to offer a platform for the development of robotic characters, typically within the context of therapeutics or entertainment. OPSORO is a unique system that provides real personalization of social robots.
\end{abstract}

Keywords - social robotics; modular; personalization; embodiment; human-robot interaction; platform

\section{INTRODUCTION}

Human-robot interaction (HRI) researchers are often faced with the choice between using an existing robotics platform, or developing their own custom robot, and each approach has its own downsides. Building a custom robot is time-consuming and expensive, but does offer a large degree of flexibility. Using existing robots is usually more feasible, but then experiments are mostly limited to the programming of the robot's behavior, the embodiment of the robot is oftentimes much harder to change. Both approaches have downsides when performing large-scale HRI experiments, where cost and flexibility are of paramount importance.

To address this issue, we have created OPSORO, a low cost open platform for the design and creation of social robots. The platfomr is specifically tailored for use with children, and designed to be produced using only do-it-yourself techniques and materials.

\section{Platform Overview}

The OPSORO platform consists of (1) a set of modules, each of which implements a facial feature, (2) electronics to drive the modules and sensors, (3) a software environment to program and control the robots, and (4) a methodology to incorporate these elements into a custom embodiment. The standard modules are combined with a custom designed skeletal frame and skin in order to easily create a unique robot.

The first robot that fully implements the OPSORO platform is Ono (Figure 1A). Ono was developed as a social robot for children. The reason we chose this target group is twofold: 1) there is a large demand for this type of robot for treatments of developmental disorders such as autism [1] and 2) other social robots (e.g. [2][3][4][5]) exist within this segment, providing us with a reference point to compare our robot to. By narrowing our focus to this application area, we gain more specific information about the usage context of the robot, which is helpful in the design process. However, we believe Ono's extensibility will allow it to be utilized within other usage contexts as well. Our choice of target audience had several consequences for the design of the robot. The entire robot is covered in a soft foam and textile skin to attain a soft and inviting appearance for children, as well as to protect the internal components from damage. The robot has a disproportionally large head to make its facial expressions more noticeable. Ono is posed in a sitting position to improve stability. As a consequence of its size and pose children can interact with the robot at eye height when the robot is placed on a table.

The platform is derived from the social robot Ono [6], [7]. And has been expanded to a new range of embodiments (see Figure 2). The design of these robots relies almost exclusively on two production techniques that are commonly found in many FabLabs: lasr cutting and 3D printing (i.e. RepRap [8] and derivatives). As far as we are aware, no other social robot platform offers the same opportunities for hacking at a similar price-point.

The complex aspects of robot embodiment design are confined within the modules, leaving the user free to design the skeleton and thus the outer appearance as they see fit. As of yet, the platform offers four types of modules: eye modules (3 DOFs), eyebrow modules (2 DOFs), mouth modules (3 DOFs), and joint modules (1 DOF). A fifth module, the neck, is currently under development. The modules of the platform focus primarily on facial features, rather than robotic limbs. This allows us to focus on face-to-face communication using low-cost hobby servos in the modules, rather than the more 

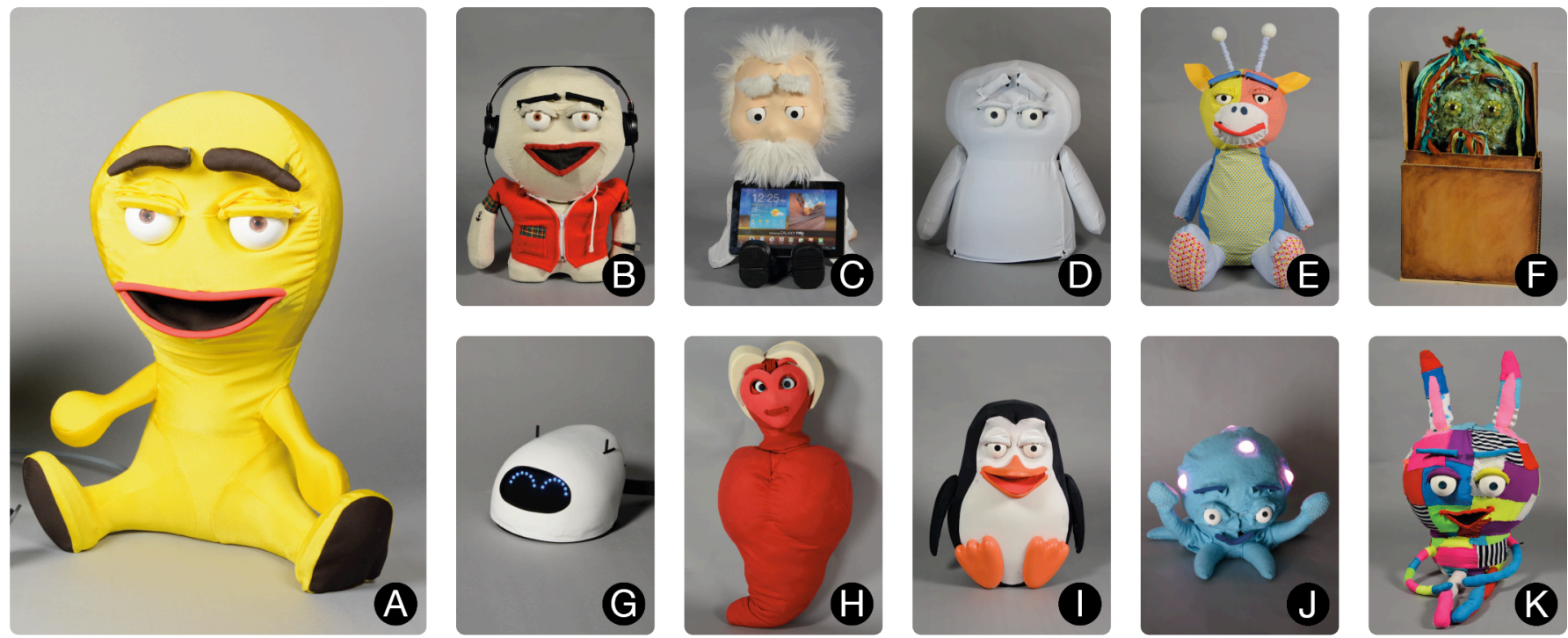

Fig 2. Overview of OPSORO robots. A: Ono, the robot from which the OPSORO platform is derived. B - K: Robots designed by industrial design students

powerful motors that would be required for limbs. This approach minimizes the production costs indicated at $300-$ 600 euro per robot.

The electronics system is based on the Raspberry Pi singleboard computer combined with a custom OPSORO daughterboard with circuitry to regulate power, control up to 16 servos, drive a speaker, read up to 12 capacitive touch sensors and 4 analog sensors. The Raspberry Pi itself has facilities for a camera input (e.g. for computer vision applications), though this is currently not yet activated in the platform. To comply with the hacking paradigm, the electronics of our platform offer many options for adaptation and extension, ranging from USB devices (e.g. a Bluetooth module or an Arduino), to I2C sensors (e.g. an accelerometer), to simple analog sensors (e.g. a button or a force-sensitive resistor).

On the software side, our platform is implemented as a web server running on the Raspberry Pi. When the board is turned on, the Wi-Fi dongle is put into access point mode and the web server is started. Users can then connect their device to the access point and control the robot through their browser. This approach allows users to operate the robot using any internetcapable device, without the need to install additional software. The interface itself borrows the "app"-metaphor of smart phones and tablets, with each task or scenario implemented as a distinct application. These apps range from simple control apps, to hardware configuration/debugging apps, to complex scripting apps. One particularly noteworthy app is the "Visual Programming" app, which lets the user implement simple scenarios using a visual programming environment. The programming environment is implemented using Blockly, a derivative of the Scratch programming language [9].

\section{ACKNOWLEDGMENT}

We would like to sincerely thank the students of the $2^{\text {nd }}$ year Industrial Design at Ghent University who participated in this project. Their enthusiasm, hard work and ingenuity made this project possible.

\section{REFERENCES}

[1] J.-J. Cabibihan, H. Javed, M. J. Ang, and S. M. Aljunied, "Why robots? A survey on the roles and benefits of social robots in the therapy of children with autism," Int. J. Soc. Robot., vol. 5, no. 4, pp. 593-618, 2013.

[2] H. Kozima, M. P. Michalowski, and C. Nakagawa, "Keepon,” Int. J. Soc. Robot., vol. 1, no. 1, pp. 3-18, Nov. 2008.

[3] K. Dautenhahn, C. L. Nehaniv, M. L. Walters, B. Robins, H. KoseBagci, N. A. Mirza, and M. Blow, "KASPAR - a minimally expressive humanoid robot for human-robot interaction research," Appl. Bionics Biomech., vol. 6, no. 3-4, pp. 369-397, Dec. 2009.

[4] J. Saldien, K. Goris, B. Vanderborght, J. Vanderfaeillie, and D. Lefeber, "Expressing emotions with the social robot Probo," Int. J. Soc. Robot., vol. 2, no. 4, pp. 377-389, 2010.

[5] G. Metta, G. Sandini, D. Vernon, L. Natale, and F. Nori, "The iCub humanoid robot," in Proceedings of the 8th Workshop on Performance Metrics for Intelligent Systems - PerMIS '08, 2008, p. 50.

[6] C. Vandevelde, J. Saldien, C. Ciocci, and B. Vanderborght, "Systems overview of Ono: a DIY reproducible open source social robot," in Lecture Notes in Computer Science, 2013, vol. 8239, pp. 311-320.

[7] C. Vandevelde, M. Vanhoucke, and J. Saldien, "Prototyping social interactions with DIY animatronic creatures," in 9th International Conference on Tangible, Embedded and Embodied Interaction, Proceedings, 2015.

[8] R. Jones, P. Haufe, E. Sells, P. Iravani, V. Olliver, C. Palmer, and A. Bowyer, "RepRap - the replicating rapid prototyper," Robotica, vol. 29, no. Special Issue 01, pp. 177-191, 2011.

[9] M. Resnick, J. Maloney, A. Monroy-Hernández, N. Rusk, E. Eastmond, K. Brennan, A. Millner, E. Rosenbaum, J. a Y. Silver, B. Silverman, and Y. Kafai, "Scratch: Programming for All.," Commun. ACM, vol. 52, pp. 60-67, 2009. 